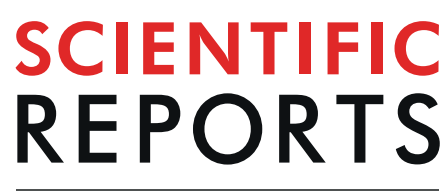

natureresearch

Check for updates

\title{
Mir34a constrains pancreatic carcinogenesis
}

\author{
Ana Hidalgo-Sastre ${ }^{1}$, Clara Lubeseder-Martellato ${ }^{1}$, Thomas Engleitner $^{2}$, Katja Steiger ${ }^{3}$, \\ Suyang Zhong ${ }^{1}$, Judit Desztics ${ }^{1}$, Rupert Öllinger ${ }^{2}$, Roland Rad ${ }^{2}$, Roland M. Schmid ${ }^{1}$, \\ Heiko Hermeking ${ }^{4,5}$, Jens T. Siveke ${ }^{6,7,8}$ \& Guido von Figura ${ }^{1 凶}$
}

Several studies have shown that over 70 different microRNAs are aberrantly expressed in pancreatic ductal adenocarcinoma (PDAC), affecting proliferation, apoptosis, metabolism, EMT and metastasis. The most important genetic alterations driving PDAC are a constitutive active mutation of the oncogene Kras and loss of function of the tumour suppressor Tp53 gene. Since the MicroRNA 34a (Mir34a) is a direct target of Tp53 it may critically contribute to the suppression of PDAC. Mir34a is epigenetically silenced in numerous cancers, including PDAC, where Mir34a down-regulation has been associated with poor patient prognosis. To determine whether Mir34a represents a suppressor of PDAC formation we generated an in vivo PDAC-mouse model harbouring pancreas-specific loss of Mir34a $\left(\operatorname{Kras}^{G 12 D} ; \mathrm{Mir34}^{\Delta / \Delta}\right)$. Histological analysis of $\mathrm{Kras}^{G 12 D} ; \mathrm{Mir34a}^{\Delta / \Delta}$ mice revealed an accelerated formation of pre-neoplastic lesions and a faster PDAC development, compared to Kras ${ }^{G 12 D}$ controls. Here we show that the accelerated phenotype is driven by an early up-regulation of the proinflammatory cytokines TNFA and IL6 in normal acinar cells and accompanied by the recruitment of immune cells. Our results imply that Mir34a restrains PDAC development by modulating the immune microenvironment of PDAC, thus defining Mir34a restauration as a potential therapeutic strategy for inhibition of PDAC development.

Pancreatic cancer is the third-leading cause of cancer-related death in the world, with a 5-year survival rate which, despite the great scientific efforts and new therapeutic technology, has only improved from $5 \%$ to $8 \%$ in the last years ${ }^{1}$. This low survival rate is the result of a combination of factors, including lack of early symptoms, lack of non-invasive detection methods and/or biomarkers, strong resistance of the tumour to chemotherapy, and a rapid metastatic spread. Surgery is the only curative option, but only a small percentage of patients qualify for resection at the time of diagnosis ${ }^{2}$.

A constitutively active form of the KRAS oncogene is the main driver mutation of pancreatic ductal adenocarcinoma (PDAC) occurring in $90 \%$ of the cases ${ }^{3}$; out of these, the substitution of G12D occurs in $41 \%$ of the cases, followed by G12V occurring in $34 \%$ of the cases and G12R in $16 \%$ of the cases ${ }^{4}$. Kras ${ }^{G 12 D}$ expression in epithelial cells leads to activation of inflammatory pathways and results in paracrine signalling with the surrounding stroma. This promotes formation and maintenance of a desmoplastic, fibro-inflammatory microenvironment, which favours the step-wise progression of normal exocrine pancreas into pre-invasive precursor acinar to ductal metaplasia (ADM) and pancreatic intraepithelial neoplasia (PanIN) lesions and PDAC development ${ }^{5}$. Additionally, loss of function of tumour suppressor genes, such as $p 53, p 16$ and SMAD4, also drives progression of the disease ${ }^{6}$.

MicroRNAs (miRNAs) are small non-coding RNAs of 20 to 25 nucleotides in length that regulate gene expression at the posttranscriptional level by binding the $3^{\prime}$-untranslated region of target mRNAs suppressing their translation and promoting their degradation ${ }^{7,8}$. Increasing evidence has shown that the expression of miRNAs is deregulated in human cancers affecting hallmark processes, such as proliferation, apoptosis, metabolism,

${ }^{1}$ Klinik und Poliklinik für Innere Medizin II, Technical University of Munich, Munich, Germany. ${ }^{2}$ Institute of Molecular Oncology and Functional Genomics, Department of Medicine II and TranslaTUM Cancer Center, Klinikum rechts der Isar, Technical University of Munich, Munich, Germany. ${ }^{3}$ Institute of Pathology, Technical University of Munich, Munich, Germany. ${ }^{4}$ Experimental and Molecular Pathology, Institute of Pathology, Ludwig Maximilian University Munich, Munich, Germany. ${ }^{5}$ German Cancer Consortium (DKTK), Partner site Munich, Munich, Germany. ${ }^{6}$ Institute for Developmental Cancer Therapeutics, West German Cancer Center, University Hospital Essen, Essen, Germany. ${ }^{7}$ Division of Solid Tumor Translational Oncology, German Cancer Consortium (DKTK, partner site Essen) and German Cancer Research Center, DKFZ, Heidelberg, Germany. ${ }^{8}$ German Cancer Consortium (DKTK), Partner site Essen, Essen, Germany. ${ }^{\circledR e}$-mail: gvfigura@tum.de 
EMT and metastasis ${ }^{9-11}$. Several microRNA encoding genes are induced by p53, among them the miR-34 gene family ${ }^{12}$. The Mir34 family is composed of Mir34a, Mir34b and Mir34c. In humans, MIR34b and c are located in chromosome 11q23.1 and transcribed from the same poly-cistronic transcript (they are located in the same exon), whereas MIR34a is encoded by a separate transcript located on chromosome 1p36.22. While MIR34b/c are mainly expressed in lung tissue ${ }^{13}$, MIR34a is ubiquitously expressed in all tissues ${ }^{14-16}$; suggesting tissue-specific functions for the different members of the Mir34 family (this is also the case in mice ${ }^{14}$ ). Members of the Mir34 family are directly activated by $\mathrm{p} 53^{14,17-19}$, among them, Mir34a is a well-known key tumour suppressor ${ }^{15,20,21}$. In cancer cells, there are two main mechanisms to inactivate tumour suppressor genes: transcriptional silencing by methylation of $\mathrm{CpG}$ islands and genomic loss. Evidence of both mechanisms have been reported for miR-34: previous studies showed how the CpG islands in the Mir34a promoter are methylated (correlating with Mir34a silencing) in different solid tumours, including pancreatic cancer ${ }^{16,22}$; and, genomic loss of the chromosomal region (1p36) of Mir34a has been reported in neuroblastoma ${ }^{20,23}$

Several studies confirmed that Mir34a is downregulated in PDAC and many other cancers (reviewed in ${ }^{10,24,25}$ ) and that it blocks tumour growth by inhibiting genes involved in various oncogenic signalling pathways. In vitro studies revealed that MIR34a is downregulated in human pancreatic cancer cells ${ }^{26}$, where it modulates Notch1 signalling, $\mathrm{Bcl} 2$, and $\mathrm{EMT}^{27-31}$.

In human PDAC patients, loss of MIR34a expression is associated with poor patient prognosis, and MIR34a levels in serum have been proposed as diagnostic biomarker for PDAC $30,32-34$. Additionally, Mir34a has a great therapeutic potential ${ }^{35}$, and it has already been tested in pre-clinical studies, where Mir34a mimics (in combination with PLK1 siRNA) was delivered using an amphiphilic nano-carrier and led to improved therapeutic response in mice $^{36}$.

Based on the above data, a tumour suppressive function of Mir34a is assumed, however, this has not been functionally tested in vivo. Here, we present an in vivo study of the tumour suppressive role of Mir34a in PDAC using genetically engineered mouse models. Mir34a is conditionally inactivated in pancreatic tissue in a $\mathrm{Kras}^{\mathrm{G} 12 \mathrm{D}}$-driven PDAC model, leading to the fast development of pre-neoplastic lesions and PDAC. This acceleration of the phenotype is driven by a cell-autonomous mechanism whereby the acinar cells generated an autocrine inflammatory response leading to recruitment of immune cells.

\section{Results}

Mir34a knockout mice rapidly develop pancreatic lesions at an early stage. To study the role of Mir34a in pancreatic carcinogenesis, the effect of conditionally knocking out Mir34a during pancreatic exocrine development was examined by crossing Mir34a $a^{f l / f l}$ mice $^{37}$, with Ptf1 $a^{+/ C r e}$ mice (hereafter called: Mir34a $a^{\Delta / \Delta}$ ) (Supplementary Fig. 1A). Since Mir34a is not expressed in normal pancreas, impairment of pancreatic exocrine development was not expected. As predicted, Mir34a $a^{\Delta / \Delta}$ mice developed normally and showed no obvious phenotype. Body weight and pancreas body weight ratio were comparable between the two groups (Supplementary Fig. 1B,C), and neither macroscopic nor histological differences were observed (Supplementary Fig. 1D,E). Furthermore, expression of pri-Mir34a in Mir34a $a^{\Delta / \Delta}$ mice was undetectable like on the WT controls (Supplementary Fig. 1F). Additionally, no compensatory effect arising from the expression of the other members of the Mir34 family (namely, Mir34b and Mir34c), was found (Supplementary Fig. 1G). Therefore, Mir34a is not required for pancreatic development, and its absence does not result in compensatory upregulation of Mir34b or Mir34c.

To study the role of Mir34a in pancreatic cancer development we crossed the Mir34a $a^{f l f l}$ mice with the well described Kras mouse model for PDAC ${ }^{38}$ to generate Ptf1a ${ }^{+/ C r e} ; \mathrm{Kras}^{+/ L S L-G 12 D}$; Mir34a $a^{f l / f l}$ mice (hereafter called: $\mathrm{Kras}^{G 12 D} ; \mathrm{Mir}^{3} 4 a^{\Delta / \Delta}$ ), which were analysed at specific time points.

At 1 month of age, the body weight and pancreas to body weight ratio were not significantly different in $\mathrm{Kras}^{G 12 D}$; Mir34a ${ }^{\Delta / \Delta}$ mice compared to controls (Supplementary Fig. 2A,B). Macroscopically, no difference between the pancreas from the two groups was observed (Fig. 1A). However, histological analysis revealed that Kras $^{G 12 D}$; Mir34a $a^{\Delta / \Delta}$ mice already presented lesions (Fig. 1A). The area of remodelled tissue (containing ADM and PanIN lesions) was quantified, and as seen by HE analysis, $\mathrm{Kras}^{G 12 D}$; Mir34a $a^{\Delta / \Delta}$ mice presented a significantly higher percentage of remodelled tissue (Fig. 1B). Interestingly, this early remodelled phenotype was conserved at 3 months of age (Fig. 1C,D) and $\mathrm{Kras}^{G 12 D}$; Mir34a $a^{\Delta / \Delta}$ mice presented macroscopically a more fibrotic pancreas (Fig. 1C). These findings were confirmed by CK19 and MUC5AC staining as markers for ADM and PanIN

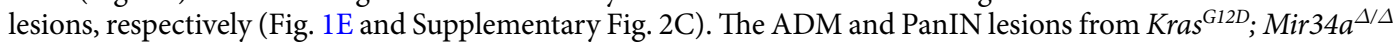
mice were not more proliferative (Supplementary Fig. 2D,E) nor apoptotic (Supplementary Fig. 2D,F) compared to those from the Kras ${ }^{G 12}$ control mice. Overall, these results show that $\mathrm{Kras}^{G 12 D}$; Mir34a $a^{\Delta / \Delta}$ mice present an acceleration in the development of ADM and PanIN pancreatic lesions, compared to Kras ${ }^{G 12 D}$ mice.

Preneoplastic lesions of $\mathrm{Kras}^{\mathrm{G12D}} ; \mathrm{Mir}^{4} \mathrm{a}^{\Delta / \Delta}$ mice progress to invasive carcinomas at 6 months of age. To gain more information about the acceleration phenotype, mice at 6 months of age were analysed. Macroscopically, the pancreas of $\mathrm{Kras}^{G 12 D}$; Mir $34 a^{\Delta / \Delta}$ mice often presented areas with a hard mass, in comparison to that of Kras ${ }^{G 12 D}$ mice which did not show macroscopic tumours (Fig. 2A). These differences were also observed histologically, the pancreas of $\mathrm{Kras}^{G 12 D}$; Mir $34 a^{\Delta / \Delta}$ mice was very fibrotic and almost lacked normal exocrine areas (Fig. 2A). Furthermore, the pancreas of $\mathrm{Kras}^{G 12 D}$; Mir34a ${ }^{\Delta / \Delta}$ mice presented significantly larger remodelled areas (Fig. 2B) with slightly more CK19 positive ADMs and significantly more MUC5A positive PanIN lesions (Fig. 2C,D). Of note, $\mathrm{Kras}^{G 12 D}$; Mir34a $a^{\Delta / \Delta}$ mice already presented significantly more invasive carcinoma (including areas with microscopic carcinomas) in comparison to Kras ${ }^{G 12 D}$ controls (Fig. 2E,F). Therefore, these results show that the preneoplastic lesions observed in Kras ${ }^{G 12 D}$; Mir $34 a^{\Delta / \Delta}$ mice at an early age are aggressive and can quickly develop into invasive carcinomas already at 6 months of age. 
A

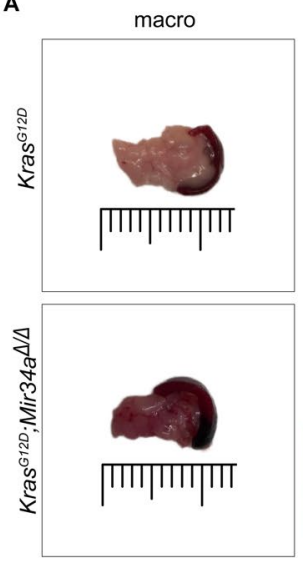

C

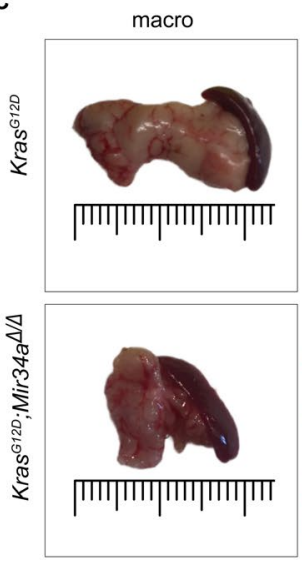

$\mathrm{HE}$
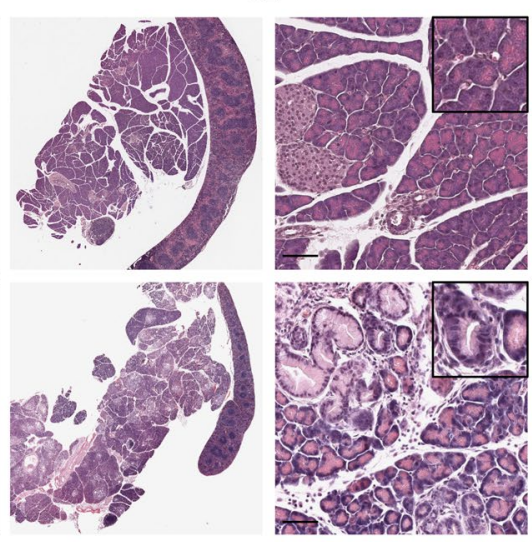

HE

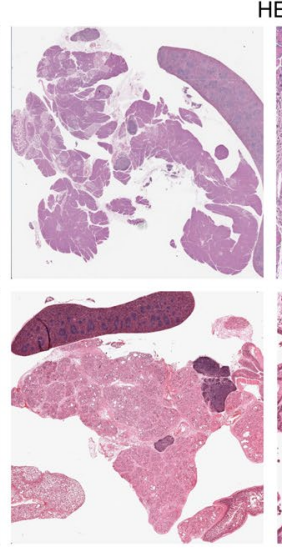

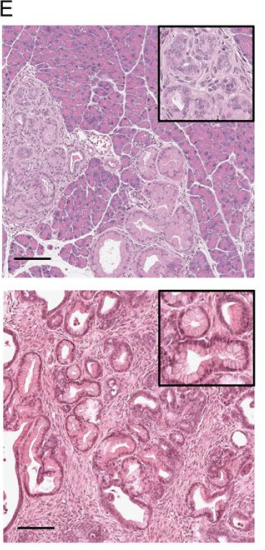

B

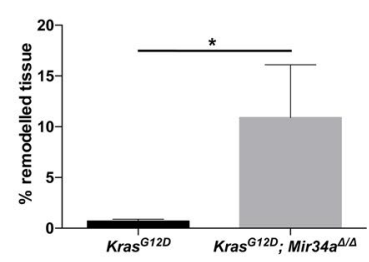

D

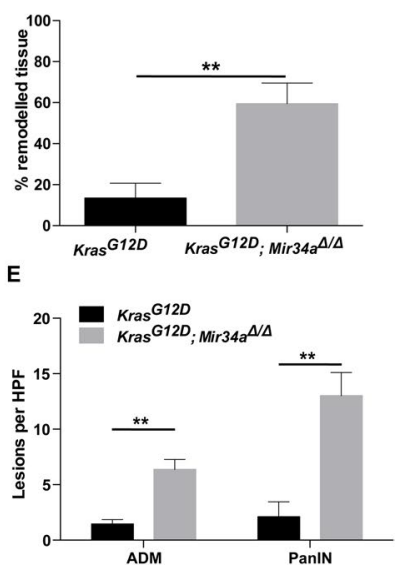

Figure 1. Mir34a knockout accelerates the development of ADM and PanIN lesions from a young age. (A)

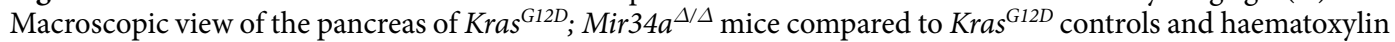
eosin (HE) staining on whole slide and a $20 \times$ zoomed in area, at 1 month of age. Scale bar $50 \mu \mathrm{m}$. (B) Quantification of the amount of pancreatic tissue remodelled (with ADM and PanIN lesions), expressed as percentage, in Kras ${ }^{G 12 D}$; Mir $34 a^{\Delta / \Delta}$ mice compared to Kras ${ }^{G 12 D}$ controls at 1 month of age ( $\mathrm{N} \geq 3$ per group). (C) Macroscopic picture and HE staining of the pancreas from $\mathrm{Kras}^{G 12 D}$; Mir34a ${ }^{\Delta / \Delta}$ mice compared to $\mathrm{Kras}^{G 12 D}$ controls at 3 months of age. Scale bar $50 \mu \mathrm{m}$. (D) Quantification of the area of pancreatic tissue remodelled shown in percentage, in $\mathrm{Kras}^{G 12 D}$; Mir34a ${ }^{\Delta / \Delta}$ mice compared to $\mathrm{Kras}^{G 12 D}$ controls at 3 months of age $(\mathrm{N} \geq 7$ per group). (E) Quantification of the amount of ADM and PanIN lesions per high power field (HPF) in Kras ${ }^{G 12 D}$; Mir34a ${ }^{\Delta / \Delta}$ mice compared to $\operatorname{Kras}^{G 12 D}$ controls ( $\mathrm{N} \geq 7$ per group).

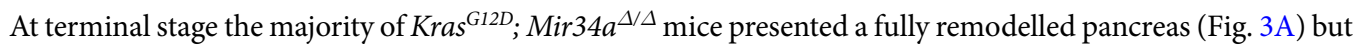
did not developed significantly more carcinomas than $\mathrm{Kras}^{G 12 D}$ mice (Fig. 3B). The lack of difference of invasive carcinoma was in agreement with the lack of increased metastasis to the lung and liver in $\mathrm{Kras}^{\text {G12D }}$; Mir34a ${ }^{\Delta / \Delta}$ (Supplementary Fig. 3A). In line with these results, the tumours of the two genotypes were macroscopically and histologically indistinguishable (Fig. 3C). Survival analysis between both groups presented a trend towards lower survival in Kras ${ }^{G 12 D}$; Mir34a $a^{\Delta / \Delta}$ mice (Supplementary Fig. 3B) supporting the acceleration phenotype hypothesis; however, the difference was not significant. Overall, these results show that the acceleration in lesion formation in $\mathrm{Kras}^{G 12 D}$; Mir34a ${ }^{\Delta / \Delta}$ mice results in higher tumour penetrance already at 6 months of age, and in a trend towards lower survival without affecting the metastatic potential.

Mir34a ablation leads to a cell-autonomous activation of inflammatory pathways. In order to gain insights into the mechanism of accelerated tumour formation and to avoid heterogeneous results due to the significant differences between the areas of normal exocrine tissue and those with pancreatic remodelling, specific cell types of $\mathrm{Kras}^{G 12 D}$; Mir34a $a^{\Delta / \Delta}$ and $\mathrm{Kras}^{G 12}$ mice at each time point were isolated. Acinar cells were isolated from pancreata of 1 month old mice (since Kras ${ }^{G 12 D} ; \mathrm{Mir}_{4} \mathrm{a}^{\Delta / \Delta}$ still present areas of normal exocrine tissue), ductal cells from remodelled pancreata of 6-month-old mice, and epithelial tumour cells were isolated from the invasive carcinomas of terminal mice. The purity of the isolated cells was assessed by gene expression analysis. All cells derived from $\mathrm{Kras}^{G 12 D}$; Mir34a ${ }^{\Delta / \Delta}$ mice did not express Mir34a (as shown by absence of pri-Mir34a mRNA expression) compared to Kras ${ }^{G 12 D}$ controls (Fig. 4A-C). Acinar cells from both $\mathrm{Kras}^{G 12 D} ; \mathrm{Mir}^{4} 4 a^{\Delta / \Delta}$ and Kras $^{G 12}$ mice, expressed comparable mRNA levels of Amylase (Fig. 4D) and did not express mRNA from the 
A
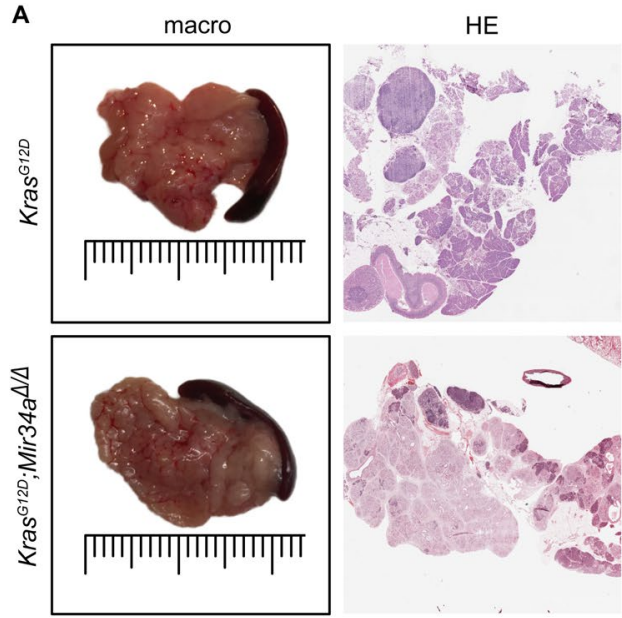

B

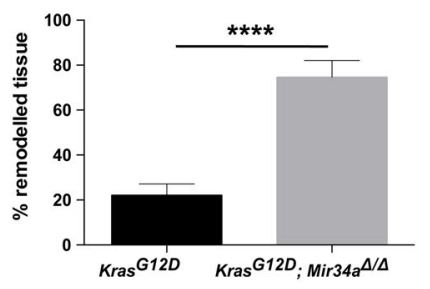

E
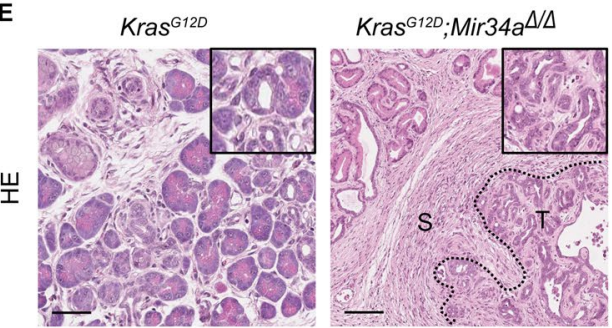

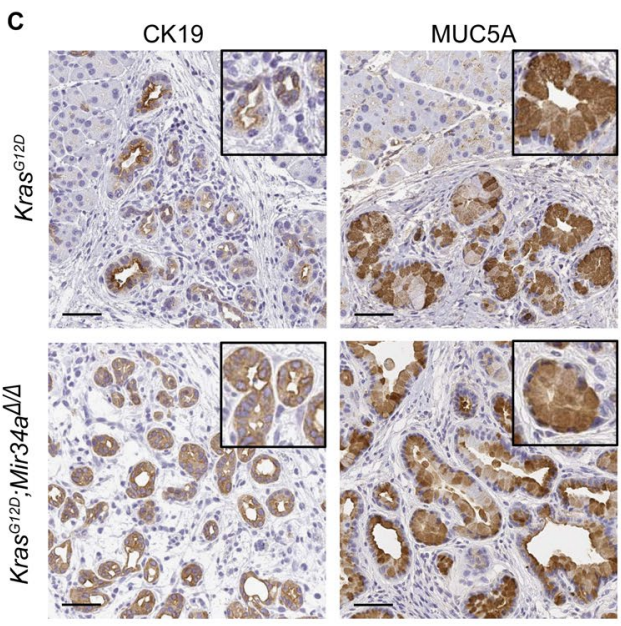

D

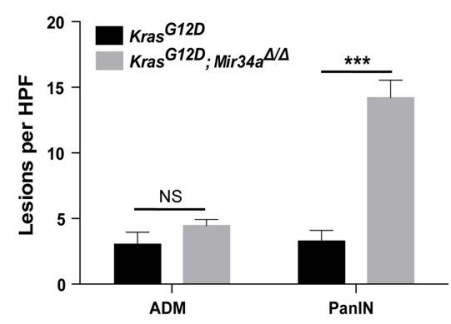

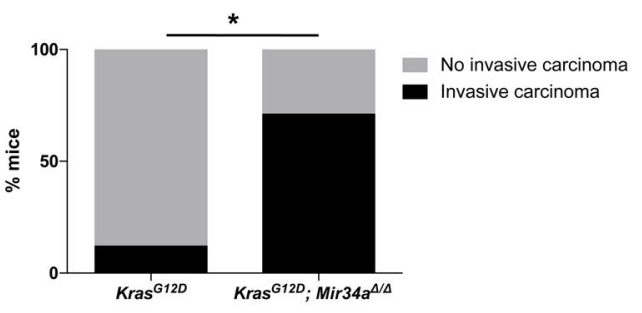

Figure 2. Mir34a knockout mice present invasive carcinomas at 6 months of age. (A) Macroscopic view of the pancreas of $\mathrm{Kras}^{G 12 D}$; Mir $34 a^{\Delta / \Delta}$ mice compared to $\mathrm{Kras}^{G 12 D}$ controls, HE staining of whole slide and a 20x zoomed in area at 6 months of age. (B) Quantification of the area, shown in percentage, of pancreatic tissue remodelled in $\mathrm{Kras}^{G 12 D}$; Mir34a ${ }^{\Delta / \Delta}$ mice compared to Kras ${ }^{G 12 D}$ controls. ( $\mathrm{N} \geq 8$ per group). (C)

Immunohistochemistry staining of the ductal marker CK19 and the PanIN marker MUC5A in Kras ${ }^{\text {G12D; }}$; Mir34a $a^{\Delta / \Delta}$ mice compared to Kras ${ }^{G 12 D}$ controls. (D) Quantification of ADM and PanIN lesions at 6 months of age in $\mathrm{Kras}^{G 12 D}$; Mir34a ${ }^{\Delta / \Delta}$ mice compared to Kras ${ }^{G 12 D}$ controls. ( $\mathrm{N} \geq 8$ per group). (E) HE staining showing an area of ADM and PanIN lesions in Kras ${ }^{G 12 D}$ mice compared to an area of PanIN lesions, stroma (S) and tumour (T) in Kras ${ }^{G 12 D}$; Mir34a ${ }^{\Delta / \Delta}$ mice. (F) Quantification of presence of invasive carcinomas, shown in percentage, in $\mathrm{Kras}^{G 12 D}$; Mir34a ${ }^{\Delta / \Delta}$ mice compared to Kras ${ }^{G 12 D}$ controls. Fisher's test $(\mathrm{N}=22)$. (A,C,E). Scale bar $50 \mu \mathrm{m}$.

ductal marker CK19 (data not shown). In contrast, the ductal cells from both $\mathrm{Kras}^{G 12 D}$; Mir34a ${ }^{\Delta / \Delta}$ and $\mathrm{Kras}^{G 12}$ mice expressed comparable mRNA levels of the ductal markers CK19 (Fig. 4E) and SOX9 (Fig. 4F) and they did not express mRNA from the acinar marker Amylase (data not shown). Therefore, the same subtype of cells was isolated in both genotypes.

In a second step, RNA sequencing and gene set enrichment analysis (GSEA) of the aforementioned cell types were performed. RNA from $\operatorname{Kras}^{G 12 D}$; Mir $34 a^{\Delta / \Delta}$ mice showed an enrichment in the following pathways: TNFA via NFKB, inflammatory response, Kras signalling up, EMT, TGFB, IL6-JAK-STAT3, across cell types compared to that of $\mathrm{Kras}^{\mathrm{G} 12}$ controls (Fig. $4 \mathrm{G}$ and Supplementary Table 1). This result suggests that acinar cells generate an autocrine inflammatory response. Furthermore, this result supports the lesion acceleration hypothesis by showing an enrichment in Kras signalling already in ductal cells at 3 months of age.

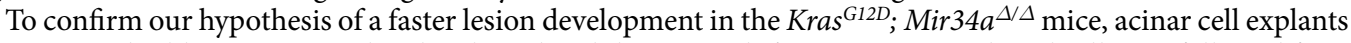
from 1-month old mice were isolated, cultured and their transdifferentiation into ductal cells was followed for 3 days (Fig. 5A). Acinar cells from Kras ${ }^{G 12 D}$; Mir $34 a^{\Delta / \Delta}$ mice showed accelerated ADM transdifferentiation rates in vitro (Fig. 5A and Supplementary Fig. 4B). Therefore, acini from Kras ${ }^{G 12 D}$; Mir $34 a^{\Delta / \Delta}$ mice transdifferentiate into ductal structures, faster than $\mathrm{Kras}^{\mathrm{G} 12}$ controls, in a cell-autonomous manner. 
A

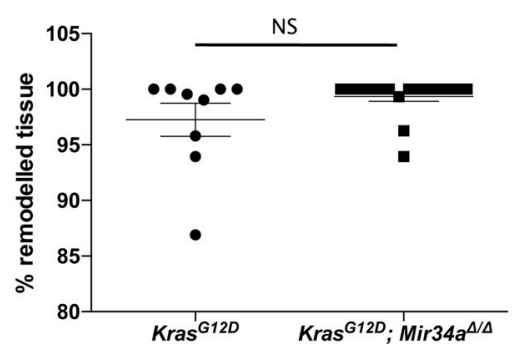

B

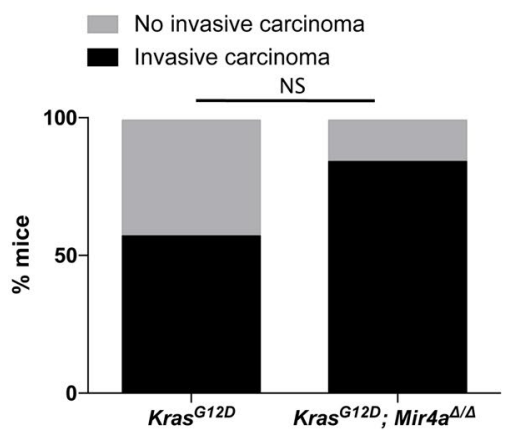

C
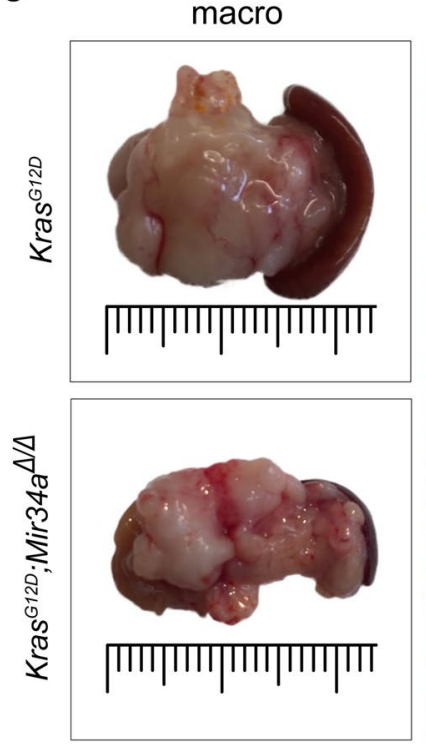

HE
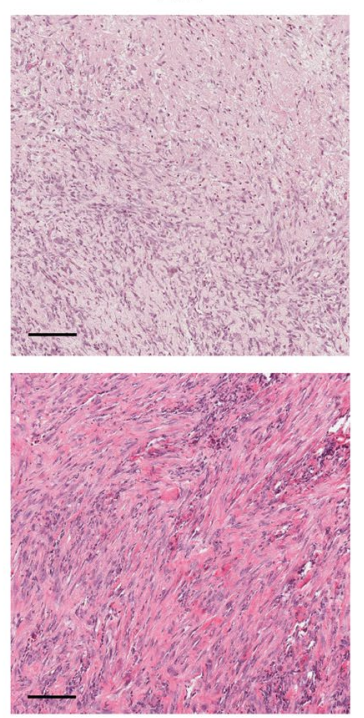

Figure 3. Mir34a knockout mice present more invasive carcinomas than Kras ${ }^{G 12 D}$ mice at terminal stage. (A) Percentage of pancreatic tissue remodelling from Kras ${ }^{G 12 D}$; Mir $34 a^{\Delta / \Delta}$ mice compared to Kras ${ }^{G 12 D}$ controls at terminal stage ( $\mathrm{N} \geq 9$ per group). (B) Quantification of presence of invasive carcinomas in $\mathrm{Kras}^{G 12 D}$; Mir34a ${ }^{\Delta / \Delta}$ mice compared to Kras ${ }^{G 12 D}$ controls, shown in percentage. Fisher's test $(\mathrm{N}=33)$ OR4 $(0.81,16.87)$. (C) Macroscopic picture of the invasive carcinoma from $\mathrm{Kras}^{G 12 D}$; Mir34a $a^{\Delta / \Delta}$ mice compared to that of $\mathrm{Kras}^{G 12 D}$ controls and HE staining of the invasive carcinoma area. Scale bar $50 \mu \mathrm{m}$.

Next, the mRNA expression levels of members of the signalling pathways enriched by GSEA were analysed in acinar cell explants. RNAs of freshly isolated acinar cell explants were analysed; expression of Tnfa, Nfkb, Il6 and Nfkbia was significantly upregulated in $\mathrm{Kras}^{G 12 D}$; Mir34a $a^{\Delta / \Delta}$ acinar cell explants (Fig. 5B). This result validates the GSEA results observed after sequencing. In summary, our results suggest that acinar cells of $\mathrm{Kras}^{\mathrm{G} 12 \mathrm{D}} ; \mathrm{Mir}^{2} 4 a^{\Delta / \Delta}$ mice develop to ADM in a cell-autonomous manner and they secrete factors which attract inflammatory cells, possibly accounting for the acceleration of invasive carcinoma development.

To confirm this hypothesis, the presence of inflammatory cells in the non-remodelled pancreas at 1 month of age was analysed (Fig. 5C). There were significantly more CD45 positive cells in $\mathrm{Kras}^{G 12 D}$; Mir34a ${ }^{\Delta / \Delta}$ mice compared to $\mathrm{Kras}^{G 12}$ controls (Fig. 5D). In addition, we validated an active TNFA and IL6 signalling in acinar cells by immunohistochemical staining of NFKB and P-STAT3 (Fig. 5E,G, respectively). The acinar cells from Kras $^{G 12 D}$; Mir34a $a^{\Delta / \Delta}$ mice had a slightly increased NFKB expression and significantly more P-STAT3 expression compared to Kras ${ }^{G 12}$ controls (Fig. 5E-H). These results demonstrate that in Kras ${ }^{G 12 D}$; Mir $34 a^{\Delta / \Delta}$ mice TNFA and IL6 expression is increased in the acinar compartment already at the age of 1 month leading to an inflammatory response and recruitment of inflammatory cells to the tissue.

\section{Discussion}

Many studies showed that plenty of microRNAs (miRNAs) are deregulated in PDAC, among them Mir34a is often downregulated and is a promising biomarker with prognostic value that correlates with diagnosis ${ }^{39-41}$. However, the exact mechanism by which Mir34a exerts its tumour suppressor role in PDAC is not clear yet.

Here we present an in vivo study where we investigated the role of Mir34a in PDAC carcinogenesis using genetically engineered mouse models. Conditional deletion of Mir34a in the pancreas of mice led to a significant acceleration in the formation of pancreatic pre-neoplastic ADM and PanINs lesions already at the age of 1 month. Furthermore, this also accelerated the formation of pancreatic invasive carcinomas, which were present already at 6 months of age. These results resemble the data from human PDAC patients in which patients with low Mir34a expression present a lower survival rate $e^{30,32-34}$. Furthermore, in agreement with our histological observations, acinar explants isolated from the pancreas of 1-month-old mice transdifferentiated faster into ductal structures in culture, suggesting a cell-autonomous mechanism. We hypothesized that the reason is that Mir34a ablation in acini from $\mathrm{Kras}^{G 12 D}$; Mir $34 a^{\Delta / \Delta}$ mice results in an alteration of signalling pathways promoting acinar differentiation. As shown by RNA sequencing and gene set enrichment analysis (GSEA), EMT, TGFB, IL6-JAK-STAT3, TNFA via NFKB, Kras signalling up, and inflammation pathways are enriched in $\mathrm{Kras}^{G 12 D}$; Mir $34 a^{\Delta / \Delta}$ mice. Additionally, the expression of Tnfa, $N f k b$ and Il6 is significantly increased, there are significantly more CD45 positive immune cells in areas of normal exocrine tissue, and significantly more NFKB and P-STAT3 accumulates in the nucleus of normal acinar cells. Based on all of these results, and since persistent inflammation in a $\mathrm{Kras}^{\mathrm{G} 12 \mathrm{D}}$ setting results in $\mathrm{PDAC}^{42}$, it is plausible that depletion of Mir34a expression drives an upregulation of 
A

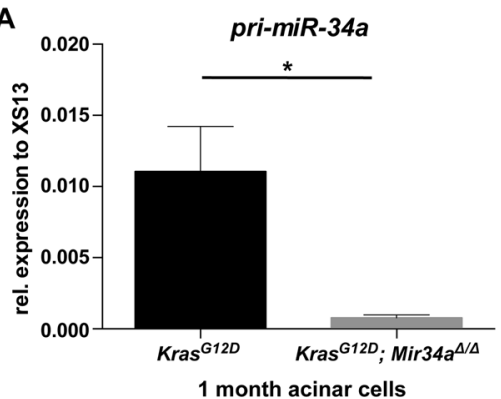

D

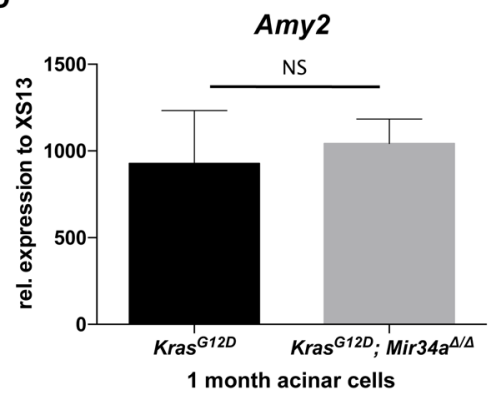

B
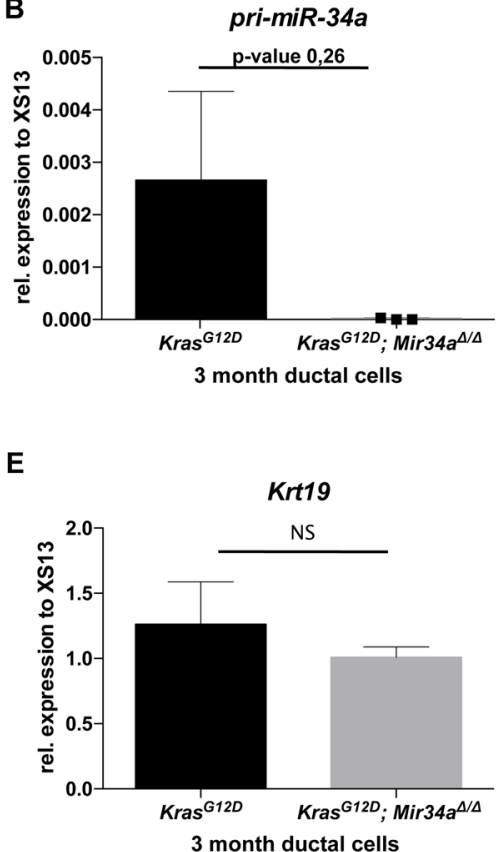

C

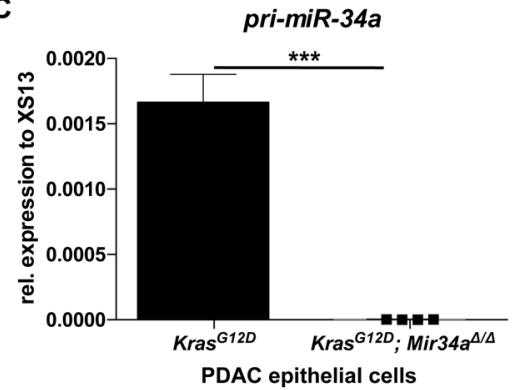

$\mathbf{F}$

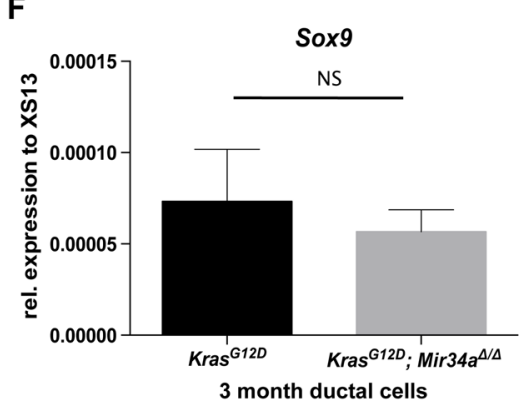

G

HALLMARK_TNFA_SIGNALING_VIA_NFKB HALLMARK_INFLAMMATORY_RESPONSE HALLMARK_KRAS_SIGNALING_UP HALLMARK_EPITHELIAL_MESENCHYMAL_TRANSITION HALLMARK_TGF_BETA_SIGNALING HALLMARK_IL6_JAK_STAT3_SIGNALING

Acinar cells Kras $^{G 12 D}$

Acinar cells Kras ${ }^{G 12 D}$; Mir34a $a^{\Delta / \Delta}$

Ductal cells Kras ${ }^{G 12 D}$

Ductal cells $K_{r a s}{ }^{G 12 D}$; Mir34a $a^{\Delta / \Delta}$

PDAC epi cells Kras ${ }^{G 12 D}$

PDAC epi cells $K_{r a s}{ }^{G 12 D} ;$ Mir34a $^{\Delta / \Delta}$

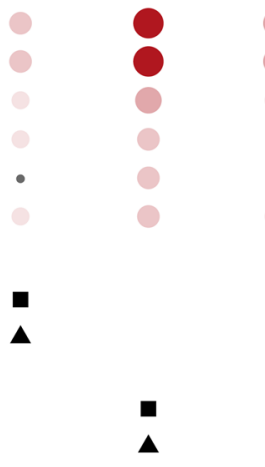

.

$\Delta$
Significant at $\alpha$ :

0.001
0.01
0.05
0.25


0.25
0.05
0.01
0.001

0.001

0.01

0.25

0.25

0.05

0.001

Figure 4. Upregulation of key signalling pathways in $\mathrm{Kras}^{G 12 D}$; Mir $34 a^{\Delta / \Delta}$ mice compared to $\mathrm{Kras}^{G 12 D}$ controls. (A) RNA expression of pri-Mir-34a in acinar explants at day 0 after isolation from $\mathrm{Kras}^{G 12 D} ; \mathrm{Mir}^{4} \mathrm{a}^{\Delta / \Delta}$ mice and $\mathrm{Kras}^{G 12 D}$ controls at 1 month of age. Welch's t-test $(\mathrm{N}=4$ per group). (B) RNA expression of pri-Mir-34a in ductal cells isolated from $\mathrm{Kras}^{G 12 D}$; Mir34a $a^{\Delta / \Delta}$ mice compared to Kras ${ }^{G 12 D}$ controls at 3 months of age. Welch's $\mathrm{t}$-test $\left(\mathrm{N}=3\right.$ per group). (C) Expression of pri-miR34a in cell lines isolated from tumour tissue of Kras ${ }^{\text {G12D. }}$; Mir34a $a^{\Delta / \Delta}$ mice compared to Kras ${ }^{G 12 D}$ controls at terminal stage. Welch's t-test ( $\mathrm{N} \geq 4$ per group). (D) RNA expression of the acinar marker Amylase in acinar explants at day 0 after isolation from Kras ${ }^{G 12 D} ; \mathrm{Mir}_{4} 4 a^{\Delta / \Delta}$ mice and $\mathrm{Kras}^{G 12 D}$ controls at 1 month of age. Welch's t-test $(\mathrm{N}=4$ per group). Differences are not statistically significant. (E,F) RNA expression of the ductal marker CK19 (E) and SOX9 (F) in ductal cells isolated from Kras $^{G 12 D}$; Mir34a ${ }^{\Delta / \Delta}$ mice compared to Kras ${ }^{G 12 D}$ controls at 3 months of age. Welch's t-test ( $=3$ per group). Differences are not significant. (G) Bubble plot showing the top 6 pathways enriched in $\mathrm{Kras}^{G 12 D}$; Mir34a $a^{\Delta / \Delta}$ mice compared to Kras ${ }^{G 12 D}$ controls after performing gene set enrichment analysis in RNA extracted from acinar cell explants at 1 month of age, ductal cells at 3 months of age, and at tumour cells at terminal stage. Red circles show upregulated pathways and blue downregulated. The size of the circle represents the p-value. Squares represent the reference group (Kras ${ }^{G 12 D}$ mice), and triangles the treatment group (Kras ${ }^{G 12 D} ; \mathrm{Mir}_{34 a^{\Delta / \Delta}}$ mice).

the inflammatory cytokines, specifically in acinar cells. This leads to NFKB and P-STAT3 activation (favouring pre-neoplastic lesion development and carcinogenesis) and to the recruitment of inflammatory cells which eventually accelerate PDAC development. In line with these findings, Mir34a was shown to negatively regulate the IL6R/STAT3 pathway in sporadic and colitis-associated colorectal cancer and thereby contribute to invasion and metastasis ${ }^{37,43}$. 

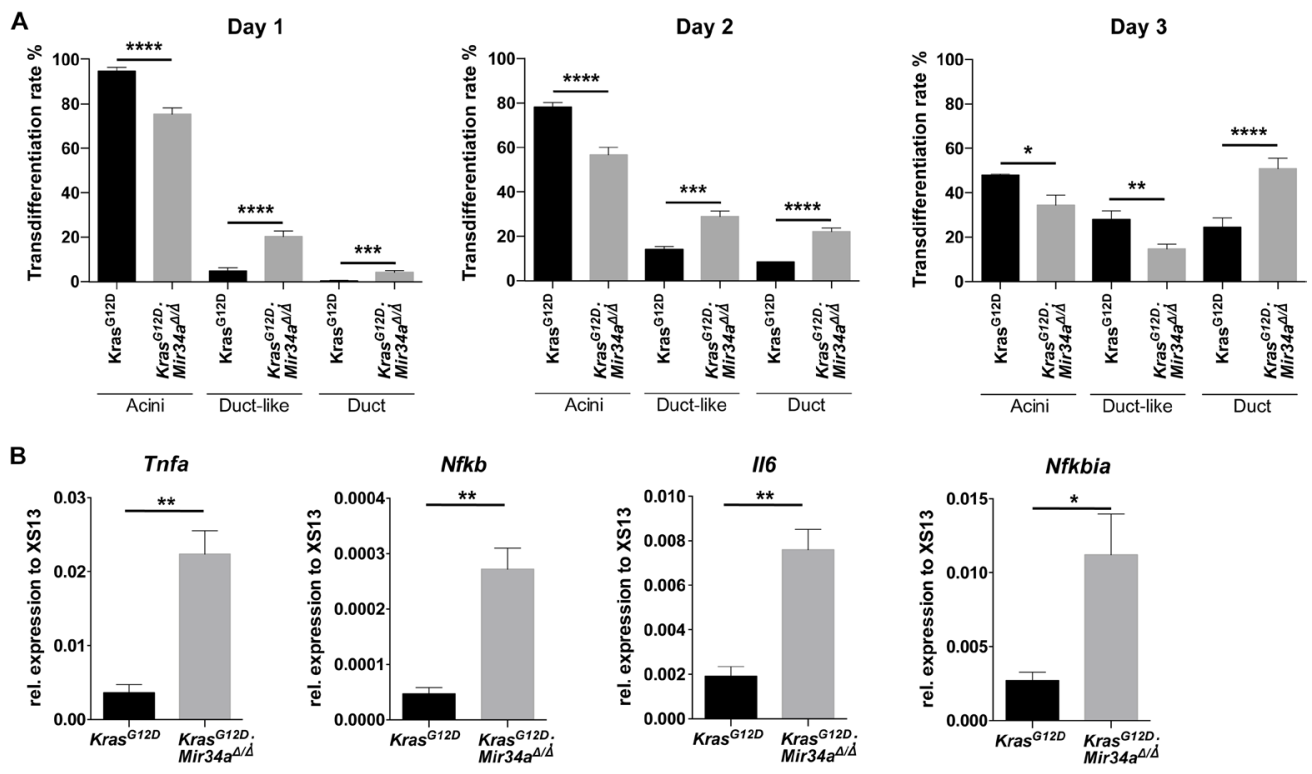

C
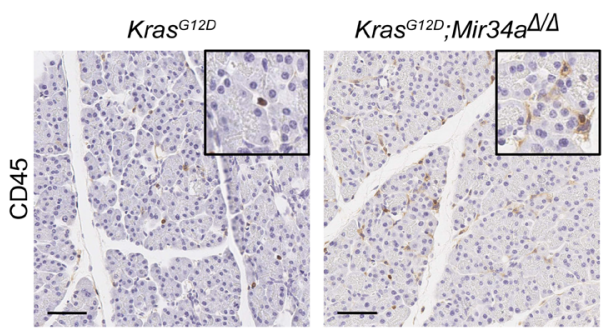

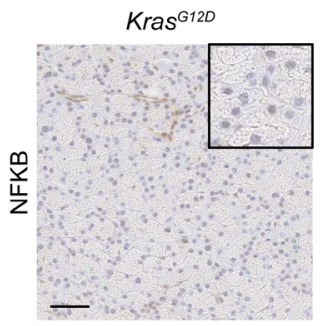

G

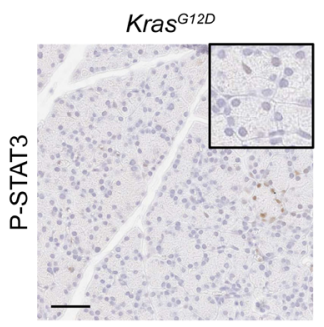

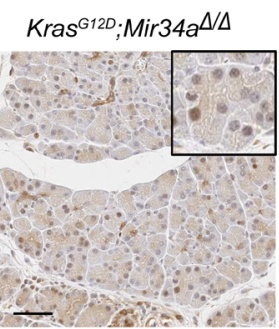

$\operatorname{Kras}^{\mathrm{G12D}} ; \mathrm{Mir}_{4} a^{\Delta / \Delta}$

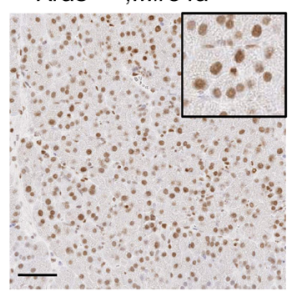

D

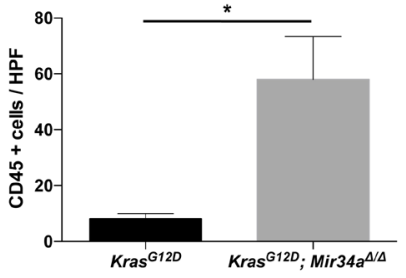

F

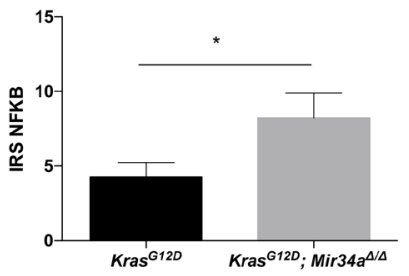

H

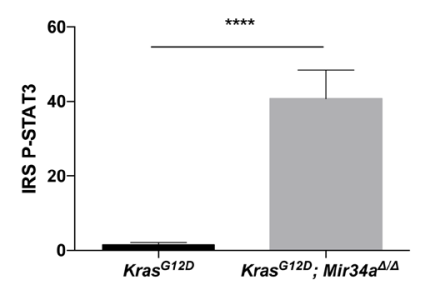

Figure 5. Mir34a knock out results in an inflammatory phenotype. (A) Average of the transdifferentiation rate of acinar cell explants from pancreata of $\mathrm{Kras}^{G 12 D}$; Mir34a $a^{\Delta / \Delta}$ and $\mathrm{Kras}^{G 12 D}$ mice into acini, duct-like and duct structures ( $\mathrm{N} \geq 3$ per group). Unpaired Student's t-test with combined SD. (B) RNA expression of members of the NFKB signalling pathway: Tnfa, $N f k b$, Il6, and $N f k b i a$, in acinar cell explants directly after isolation (day 0) from Kras ${ }^{G 12 D}$;

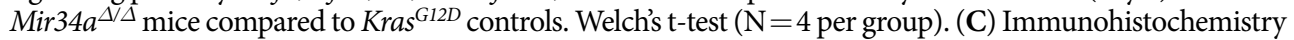
staining for CD45 in tissue from $\mathrm{Kras}^{G 12 D}$; Mir34a ${ }^{\Delta / \Delta}$ mice compared to Kras ${ }^{G 12 D}$ controls. Scale bar $50 \mu \mathrm{m}$. (D) Quantification of CD45 positive cells per high power field in areas of normal tissue from $\mathrm{Kras}^{G 12 D}$; $\mathrm{Mir}^{\mathrm{B}} 4 \mathrm{a}^{\Delta / \Delta}$ mice compared to $K r a s^{G 12 D}$ controls ( $\mathrm{N} \geq 3$ per group). (E) Immunohistochemistry staining for NFKB in tissue from

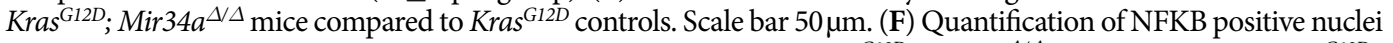
from acinar cells in areas of normal tissue per high power field from $\mathrm{Kras}^{G 12 D}$; Mir34a ${ }^{\Delta / \Delta}$ mice compared to $\mathrm{Kras}^{G 12 D}$ controls ( $\mathrm{N} \geq 3$ per group). (G) Immunohistochemistry staining for P-STAT3 in tissue from $\mathrm{Kras}^{G 12 D} ; \mathrm{Mir}^{\mathrm{M}} 4 \mathrm{a}^{\Delta / \Delta}$ mice compared to Kras ${ }^{G 12 D}$ controls. Scale bar $50 \mu \mathrm{m}$. (H) Quantification of P-STAT3 positive nuclei from acinar cells in areas of normal tissue per high power field from Kras ${ }^{G 12 D} ; M_{i r 34 a^{\Delta / \Delta}}$ mice compared to Kras ${ }^{G 12 D}$ controls $(\mathrm{N} \geq 3$ per group). 
MicroRNAs (mRNAs), including Mir34a, were reported to be key inflammation regulators (reviewed in ${ }^{44}$ ). The signalling pathways enriched by GSEA in $\mathrm{Kras}^{\mathrm{G12D}}$; Mir34a ${ }^{\Delta / \Delta}$ mice, are inflammatory pathways strongly related between themselves and their upregulation was in line with the literature. One of the signalling pathways enriched at early time points was TGFB. A recent study with mouse models, revealed that activation of TGFB pathway during early pancreatic tumorigenesis induces ADM reprogramming, by activating apoptosis and cell differentiation and provides a favourable environment for the development of $\mathrm{Kras}^{\mathrm{G} 12 \mathrm{D}}$-driven preneoplastic lesions and carcinogenesis ${ }^{45}$. This was also confirmed in human pancreatic cells ${ }^{46,47}$. Furthermore, SMAD4, the main effector of the TGFB signalling pathway is also a direct target of Mir34 $a^{48-50}$. Additionally, IL6-JAK-STAT3, TNFA via NFKB and inflammation pathways are also enriched by GSEA and we show that ablation of Mir34a in $\mathrm{Kras}^{G 12 D}$; Mir34a ${ }^{\Delta / \Delta}$ mice led to an increase in TNFA and IL6 in pancreatic acini. Multiple studies using mouse models of pancreatic cancer demonstrate a strong relationship between Kras, NFKB, STAT3 and cytokine signalling which drives lesion formation and the development of PDAC ${ }^{51-57}$.

Our results are in agreement with previous studies showing that Mir34a downregulates TNFA and IL6 ${ }^{58}$. For instance, in vitro administration of Mir34a mimics to LPS treated macrophages decreases the expression of TNFA and IL6, and reduces NFKB activation. Here the mechanism shown is direct inhibition of Mir34a over either its target Notch1, which activates the inflammatory response in macrophages, or other genes that affect NFKB signalling; therefore, modulating LPS-induced macrophage inflammatory response ${ }^{58}$. Furthermore, in colorectal cancer Mir34a was shown to constrain carcinogenesis by directly inhibiting an IL-6R/STAT3/Mir34a feedback loop, and its ablation is required to induce IL6-mediated EMT and invasion ${ }^{37}$. Moreover, a couple of studies support that IL-6R is a direct target of Mir34a $a^{43,59,60}$. Therefore, many previous studies have reported similar results to ours in other organs and supports our observation that Mir34a ablation results in the upregulation of the inflammatory cytokines TNFA and IL6 in the pancreas. Interestingly, these two secreted pro-inflammatory cytokines are considered strong EMT inducers key for cancer progression ${ }^{61}$. However, in our study despite that an enrichment in EMT signature was found at early time points, it was not sufficient to result in significantly more metastasis in $\mathrm{Kras}^{G 12 D}$; Mir34a $a^{\Delta / \Delta}$ mice, possibly due to the fact that at later stages of the disease Kras ${ }^{G 12}$ mice also present a strong EMT signature or due to the faster tumour development.

In summary, this study shows that ablation of Mir34a results in a cell-autonomous inflammatory response marked by the increase in TNFA and IL6 expression in acinar cells, which is linked to an enhanced TGFB signalling in preneoplastic transformation that appears to accelerate $\mathrm{Kras}^{G 12 D}$-dependent pancreatic carcinogenesis. Overall, our results suggest that apart from its known tumor suppressor role, Mir34a also has an anti-inflammatory role in the pancreas by downregulating TNFA and IL6. This anti-inflammatory role may be important at the initiation stage of preneoplastic development, but further functional understanding of the underlying mechanisms is required in order to fully support this hypothesis. This capacity of Mir $34 a$ should also be taken into account when designing Mir34a targeted therapy for PDAC.

\section{Methods}

Mouse strains. Mir $34 a^{f l / f l}$ mice ${ }^{37}$ were generated by the Hermeking laboratory and we bred them to Ptfla $a^{+1}$ Cre 62 and $\mathrm{Kras}^{+/ L S L-G 12 D} 38$ mice to generate Ptf1 $a^{+/ C r e}$; Mir34a $a^{f l f l}$ (called: Mir34a ${ }^{\Delta / \Delta}$ ) and Ptf1 $a^{+/ C r e}$; Kras ${ }^{+/ L S L-}$ G12D; Mir34a $a^{f l f l}$ (called: Kras ${ }^{G 12 D} ;$ Mir34a $a^{\Delta / \Delta}$ ). Co-housed wild type Mir34a $a^{f l / f l}$ and Ptf1 $a^{+/ C r e}$; Kras ${ }^{+/ L S L-G 12 D}$ (called: $\mathrm{Kras}^{G 12 D}$ ) littermate mice were used as controls. All animal experiments were conducted in accordance with German Federal Animal Protection Laws and approved by the Institutional Animal Care and Use Committee from the Technical University of Munich (Germany), and from the Government of Bavaria AZ: (5.2-1-54-2532-46-2014).

Body and pancreatic weight analysis. Mice were sacrificed and immediately weighted in a scale. Subsequently, the whole pancreas was carefully excised without any adjacent tissue and directly weighed in a precision scale under sterile technique.

Primary cell isolation and culture. After sacrificing the mice, either acinar, ductal or tumour cells (from terminal mice) were directly isolated from the pancreas and cultured using the methods previously described ${ }^{63,64}$. For quantification of acinar explants transdifferentiation rate, acinar cell explants were counted in 6 to 33 high power fields per mouse using a 10X objective (approx. 3 areas per well of a 48 well plate). The phenotype was defined as acinar (cluster of acinar cells resembling a ball), duct-like (acinar cell clusters with a ductal structure emerging within the cluster) or ductal (ductal structure with a defined lumen), see Supplementary Fig. 4A.

Immunohistochemical staining. Immunohistochemistry was performed as previously described ${ }^{65}$. The following primary antibodies were used: rabbit anti-Cleaved caspase 3 (1:200; Cell Signalling \#9661), mouse anti-CD45 (1:20, BDPharmigen \#550539), rabbit anti-CK19 (1:1000; Abcam \#ab133496), goat anti-CPA1 (1:300, RD Systems \#AF2765), mouse anti-Ki-67 (1:400; BDPharmigen \#550609), mouse anti-MUC5AC (1:200; Cell Marque \#292M-95), rabbit anti-p65 (C-20) (1:200, Santa Cruz \#sc-372), rabbit anti-phospho-STAT3 (Y705) (1:100, Cell Signaling \#9145).

Morphometric quantification. After staining, slides were scanned at 20x using a Leica AT2 scanner (Leica) and analysed with Aperio Image Scope program (Leica). Whole pancreatic area and remodelled tissue (areas of $\mathrm{ADM}$ and/or low/high grade PanIN lesions) were quantified in a blinded way; representative images were taken. For quantification of CK19 positive ADM and MUC5AC-positive PanIN lesions, 10 high power fields (HPF) were counted per whole pancreatic tissue slide and the average number of lesions per HPF was calculated. For quantification of Ki-67 and cleaved caspase 3, $50 \mathrm{ADM}$ and PanIN lesions were randomly selected across the whole slide (no more than 10 lesions in the same area), positive cells (visualized by brown precipitate) were counted and the percentage was calculated. The total number of cells counted per HPF excluded: fatty, edema, or inflammatory 


\begin{tabular}{|c|c|c|}
\hline Gene & Primer & Sequence $\left(5^{\prime}-3^{\prime}\right)$ \\
\hline \multirow{2}{*}{ Mir34a } & Pri-miR-34a_F & 5'-CTGTGCCCTCTTGCAAAAGG-3' \\
\hline & Pri-miR-34a_R & 5'-GGACATTCAGGTGAGGGTCTTG-3' \\
\hline \multirow{2}{*}{ Mir34bc } & Pri-miR-34bc_F & 5'-GGCAGGAAGGCTCCAGATG-3' \\
\hline & Pri-miR-34bc_R & 5'-CCTCACTGTTCATATGCCCATTC-3' \\
\hline \multirow{2}{*}{ Amylase } & Amy2a_F & 5'-TGGTCAATGGTCAGCCTTTTTC-3' \\
\hline & Amy2a_R & 5'-CACAGTATGTGCCAGCAGGAAG-3' \\
\hline \multirow{2}{*}{ CK19 } & Krt19_F & 5'-ACCCTCCCGAGATTACAACC-3' \\
\hline & Krt19_R & 5'-CAAGGCGTGTTCTGTCTCAA-3' \\
\hline \multirow{2}{*}{ SOX9 } & SOX9_F & 5'-CCACGTGTGGATGTCGAAG- $3^{\prime}$ \\
\hline & SOX9_R & 5'-CTCAGCTGCTCCGTCTTGAT-3' \\
\hline \multirow{2}{*}{ Tnfa } & Tnfa_F & 5'-TGCCTATGTCTCAGCCTCTTC-3' \\
\hline & Tnfa_R & 5'-GAGGCCATTTGGGAACTTCT-3' \\
\hline \multirow{2}{*}{$N f k b$} & $N f k b_{-} \mathrm{F}$ & 5'-GGAGGCATGTTCGGTAGTGG-3' \\
\hline & Nfkb_R & 5'-CCCTGCGTTGGATTTCGTG-3' \\
\hline \multirow{2}{*}{ Il6 } & Il6_F & 5'-GCTACCAAACTGGATATAATCAGGA-3' \\
\hline & Il6_R & 5'-CCAGGTAGCTATGGTACTCCAGAA - $3^{\prime}$ \\
\hline \multirow{2}{*}{ Nfkbia } & Nfkbia_F & 5'-TGAAGGACGAGGAGTACGAGC-3' \\
\hline & Nfkbia_R & 5'-TCTTCGTGGATGATTGCCAAG-3' \\
\hline \multirow{2}{*}{ XS13 } & XS13_F & 5'-TGGGCAAGAACACCATGATG-3' \\
\hline & XS13_R & 5'-AGTTTCTCCAGAGCTGGGTTGT-3' \\
\hline
\end{tabular}

Table 1. Primers used for RT-PCR.

tissue. Presence of microscopic carcinomas (only a few cancer cells) and pancreatic ductal adenocarcinoma was determined by an experienced pathologist (K.S.). For the quantification of CD45 positive immune cells, $10 \mathrm{HPF}$ were randomly selected across the whole pancreatic tissue slide in areas of normal tissue. Only positive immune cells were counted. For the quantification of NFKB and P-STAT3 positive nuclei the nuclear counting algorithm V9 from Image Scope was used with the following settings: threshold for cytoplasmic correction 230, upper limit of weak $(1+) 217$, moderate $(2+) 200$ and strong $(3+) 188.10$ HPF were randomly selected across the whole pancreatic tissue section in areas of normal tissue. Only nuclei from normal acini were counted.

RNA isolation and quantitative RT-PCR. After sacrificing the mice, a small piece of the pancreas was excised, stored in RNAlater (Qiagen) overnight at $4^{\circ} \mathrm{C}$ and stored at $-80^{\circ} \mathrm{C}$ the next morning. RNA was homogenized with RA1 lysis buffer (Macherey-Nagel) and $\beta$-mercaptoethanol. Subsequently, RNA was isolated using the Maxwell 16 LEV simplyRNA Purification Kit (Promega) following manufacturer's instructions. SuperScript II Reverse Transcriptase (Invitrogen) was used for cDNA synthesis, according to manufacturer's protocol. RT-PCR was performed as previously described ${ }^{65}$ using the SYBR Green master mix (Roche) on a Lightcycler 480 system (Roche). Primers used are described in Table 1. Melting curve analysis was performed to ensure product quality and specificity. Expression levels of each transcript were normalized to the housekeeping gene XS13 (a constitutively expressed ribosomal protein with same levels in normal, cancerous, and inflamed human pancreas ${ }^{66}$, using the $\Delta \Delta \mathrm{Ct}$ method. All RT-PCR experiments were performed with at least $\mathrm{N}=3$ individual biological samples per group.

RNA-sequencing. Library preparation for bulk $3^{\prime}$-sequencing of poly(A)-RNA was done as described previously ${ }^{67}$. Briefly, barcoded cDNA of each sample was generated with a Maxima RT polymerase (Thermo Fisher) using oligo-dT primer containing barcodes, unique molecular identifiers (UMIs) and an adapter. $5^{\prime}$ ends of the cDNAs were extended by a template switch oligo (TSO) and after pooling of all samples full-length cDNA was amplified with primers binding to the TSO-site and the adapter. cDNA was tagmented with the Nextera XT kit (Illumina) and $3^{\prime}$-end-fragments finally amplified using primers with Illumina P5 and P7 overhangs. The library was sequenced on a NextSeq. 500 (Illumina) with 16 cycles for the barcodes and UMIs in read 1 and 65 cycles for the cDNA in read2.

RNAseq analysis. Gencode gene annotations version M18 and the mouse reference genome major release GRCm38 were derived from the Gencode homepage (https://www.gencodegenes.org/). Dropseq tools v1.12 ${ }^{68}$ was used for mapping the raw sequencing data to the reference genome. The resulting UMI filtered countmatrix was imported into R v3.4.4. Prior differential expression analysis with DESeq. $21.18 .1^{69}$, dispersion of the data was estimated with a parametric fit. The Wald test was used for determining differentially regulated genes between experimental conditions and shrunken $\log 2$ fold changes were calculated afterwards, with setting the type argument of the lfcShrink function to 'normal'.

A gene was determined to be differentially regulated if the adjusted p-value was below 0.05 . Gene set enrichment analysis was conducted with the preranked GSEA method ${ }^{70}$ within the MSigDB Hallmark database. Genes were ranked according to their respective $\log 2$ fold change. A pathway was considered to be significantly associated with an experimental condition at an alpha level of 0.05 (for NES and FDR values see Supplementary Table 1). 
Statistical analysis. Statistical analysis was performed using Graph Pad Prism6 (GraphPad Software Inc). Unless otherwise stated, the Mann-Whitney Test for non-normal distributed unpaired data was used for inter-group comparison. For Fischer's test OR (95\% CI) was used. Kaplan-Meier curve was calculated using the survival time for each mouse from the littermate groups. The log-rank test was used to address significant differences between the groups. For RT-PCR data, Log2 values were used for conducting the t-test. Welch's t-test was used when samples followed a Gaussian distribution but they had different standard deviations. For all statistical analysis, differences with a p-value lower than 0.05 were considered significant, and the following scale was applied: $* \mathrm{p}<0.05, * * \mathrm{p}<0.01, * * * \mathrm{p}<0.001, * * * * \mathrm{p}<0.0001$. Data are presented as mean values \pm SEM, unless otherwise stated.

Received: 2 February 2020; Accepted: 18 May 2020;

Published online: 15 June 2020

\section{References}

1. Siegel, R. L., Miller, K. D. \& Jemal, A. Cancer statistics, 2018. CA Cancer J Clin 68, 7-30, https://doi.org/10.3322/caac.21442 (2018).

2. Zhu, H., Li, T., Du, Y. \& Li, M. Pancreatic cancer: challenges and opportunities. BMC Med 16, 214, https://doi.org/10.1186/s12916018-1215-3 (2018).

3. Almoguera, C. et al. Most human carcinomas of the exocrine pancreas contain mutant c-K-ras genes. Cell 53, 549-554 (1988)

4. Waters, A. M. \& Der, C. J. KRAS: The Critical Driver and Therapeutic Target for Pancreatic Cancer. Cold Spring Harb Perspect Med 8, https://doi.org/10.1101/cshperspect.a031435 (2018).

5. Collins, M. A. et al. Oncogenic Kras is required for both the initiation and maintenance of pancreatic cancer in mice. J Clin Invest 122, 639-653, https://doi.org/10.1172/JCI59227 (2012).

6. Hruban, R. H., Goggins, M., Parsons, J. \& Kern, S. E. Progression model for pancreatic cancer. Clin Cancer Res 6, 2969-2972 (2000).

7. Bhardwaj, A., Arora, S., Prajapati, V. K., Singh, S. \& Singh, A. P. Cancer "stemness"- regulating microRNAs: role, mechanisms and therapeutic potential. Curr Drug Targets 14, 1175-1184 (2013).

8. Srivastava, S. K. et al. MicroRNAs as potential clinical biomarkers: emerging approaches for their detection. Biotech Histochem 88, 373-387, https://doi.org/10.3109/10520295.2012.730153 (2013).

9. Srivastava, S. K. et al. MicroRNAs in pancreatic malignancy: progress and promises. Cancer Lett 347, 167-174, https://doi. org/10.1016/j.canlet.2014.02.015 (2014).

10. Misso, G. et al. Mir-34: a new weapon against cancer? Mol Ther Nucleic Acids 3, e194, https://doi.org/10.1038/mtna.2014.47 (2014).

11. Rokavec, M., Li, H., Jiang, L. \& Hermeking, H. The p53/miR-34 axis in development and disease. J Mol Cell Biol 6, 214-230, https:// doi.org/10.1093/jmcb/mju003 (2014).

12. Hermeking, H. MicroRNAs in the p53 network: micromanagement of tumour suppression. Nat Rev Cancer 12, 613-626, https://doi. org/10.1038/nrc3318 (2012).

13. Liang, Y., Ridzon, D., Wong, L. \& Chen, C. Characterization of microRNA expression profiles in normal human tissues. BMC Genomics 8, 166, https://doi.org/10.1186/1471-2164-8-166 (2007).

14. Bommer, G. T. et al. p53-mediated activation of miRNA34 candidate tumor-suppressor genes. Curr Biol 17, 1298-1307, https://doi. org/10.1016/j.cub.2007.06.068 (2007).

15. Hermeking, H. The miR-34 family in cancer and apoptosis. Cell Death Differ 17, 193-199, https://doi.org/10.1038/cdd.2009.56 (2010).

16. Lodygin, D. et al. Inactivation of miR-34a by aberrant CpG methylation in multiple types of cancer. Cell Cycle 7, 2591-2600, https:// doi.org/10.4161/cc.7.16.6533 (2008).

17. He, L. et al. A microRNA component of the 553 tumour suppressor network. Nature 447, 1130-1134, https://doi.org/10.1038/ nature05939 (2007).

18. Raver-Shapira, N. et al. Transcriptional activation of miR-34a contributes to p53-mediated apoptosis. Mol Cell 26, 731-743, https:// doi.org/10.1016/j.molcel.2007.05.017 (2007)

19. Tarasov, V. et al. Differential regulation of microRNAs by 533 revealed by massively parallel sequencing: miR-34a is a p 53 target that induces apoptosis and G1-arrest. Cell Cycle 6, 1586-1593, https://doi.org/10.4161/cc.6.13.4436 (2007).

20. Cole, K. A. et al. A functional screen identifies miR-34a as a candidate neuroblastoma tumor suppressor gene. Mol Cancer Res 6, 735-742, https://doi.org/10.1158/1541-7786.MCR-07-2102 (2008).

21. Bagchi, A. \& Mills, A. A. The quest for the 1 p36 tumor suppressor. Cancer Res 68, 2551-2556, https://doi.org/10.1158/0008-5472. CAN-07-2095 (2008).

22. Vogt, M. et al. Frequent concomitant inactivation of miR-34a and miR-34b/c by CpG methylation in colorectal, pancreatic, mammary, ovarian, urothelial, and renal cell carcinomas and soft tissue sarcomas. Virchows Arch 458, 313-322, https://doi. org/10.1007/s00428-010-1030-5 (2011).

23. Attiyeh, E. F. et al. Chromosome 1p and 11q deletions and outcome in neuroblastoma. N Engl J Med 353, 2243-2253, https://doi. org/10.1056/NEJMoa052399 (2005).

24. Li, X. J., Ren, Z. J. \& Tang, J. H. MicroRNA-34a: a potential therapeutic target in human cancer. Cell Death Dis 5, e1327, https://doi. org/10.1038/cddis.2014.270 (2014).

25. Slabakova, E., Culig, Z., Remsik, J. \& Soucek, K. Alternative mechanisms of miR-34a regulation in cancer. Cell Death Dis 8, e3100, https://doi.org/10.1038/cddis.2017.495 (2017).

26. Chang, T. C. et al. Transactivation of miR-34a by p53 broadly influences gene expression and promotes apoptosis. Mol Cell 26, 745-752, https://doi.org/10.1016/j.molcel.2007.05.010 (2007).

27. Xia, J. et al. Genistein inhibits cell growth and induces apoptosis through up-regulation of miR-34a in pancreatic cancer cells. Curr Drug Targets 13, 1750-1756 (2012).

28. Ji, Q. et al. MicroRNA miR-34 inhibits human pancreatic cancer tumor-initiating cells. PLoS One 4, e6816, https://doi.org/10.1371/ journal.pone.0006816 (2009).

29. Tang, Y., Tang, Y. \& Cheng, Y. S. miR-34a inhibits pancreatic cancer progression through Snaill-mediated epithelial-mesenchymal transition and the Notch signaling pathway. Sci Rep 7, 38232, https://doi.org/10.1038/srep38232 (2017).

30. Long, L. M. et al. The Clinical Significance of miR-34a in Pancreatic Ductal Carcinoma and Associated Molecular and Cellular Mechanisms. Pathobiology 84, 38-48, https://doi.org/10.1159/000447302 (2017).

31. Ikeda, Y., Tanji, E., Makino, N., Kawata, S. \& Furukawa, T. MicroRNAs associated with mitogen-activated protein kinase in human pancreatic cancer. Mol Cancer Res 10, 259-269, https://doi.org/10.1158/1541-7786.MCR-11-0035 (2012).

32. Alemar, B. et al. miRNA-21 and miRNA-34a Are Potential Minimally Invasive Biomarkers for the Diagnosis of Pancreatic Ductal Adenocarcinoma. Pancreas 45, 84-92, https://doi.org/10.1097/MPA.0000000000000383 (2016).

33. Jamieson, N. B. et al. MicroRNA molecular profiles associated with diagnosis, clinicopathologic criteria, and overall survival in patients with resectable pancreatic ductal adenocarcinoma. Clin Cancer Res 18, 534-545, https://doi.org/10.1158/1078-0432.CCR$11-0679$ (2012) 
34. Ohuchida, K. et al. MicroRNA expression as a predictive marker for gemcitabine response after surgical resection of pancreatic cancer. Ann Surg Oncol 18, 2381-2387, https://doi.org/10.1245/s10434-011-1602-x (2011).

35. Bader, A. G. miR-34 - a microRNA replacement therapy is headed to the clinic. Front Genet 3, 120, https://doi.org/10.3389/ fgene.2012.00120 (2012).

36. Gibori, H. et al. Amphiphilic nanocarrier-induced modulation of PLK1 and miR-34a leads to improved therapeutic response in pancreatic cancer. Nat Commun 9, 16, https://doi.org/10.1038/s41467-017-02283-9 (2018).

37. Rokavec, M. et al. IL-6R/STAT3/miR-34a feedback loop promotes EMT-mediated colorectal cancer invasion and metastasis. J Clin Invest 124, 1853-1867, https://doi.org/10.1172/JCI73531 (2014).

38. Hingorani, S. R. et al. Preinvasive and invasive ductal pancreatic cancer and its early detection in the mouse. Cancer Cell 4, 437-450 (2003).

39. Guo, S., Fesler, A., Wang, H. \& Ju, J. microRNA based prognostic biomarkers in pancreatic Cancer. Biomark Res 6, 18, https://doi. org/10.1186/s40364-018-0131-1 (2018).

40. Slotwinski, R., Lech, G. \& Slotwinska, S. M. MicroRNAs in pancreatic cancer diagnosis and therapy. Cent Eur J Immunol 43, 314-324, https://doi.org/10.5114/ceji.2018.80051 (2018).

41. Ren, L. \& Yu, Y. The role of miRNAs in the diagnosis, chemoresistance, and prognosis of pancreatic ductal adenocarcinoma. Ther Clin Risk Manag 14, 179-187, https://doi.org/10.2147/TCRM.S154226 (2018).

42. Kitajima, S., Thummalapalli, R. \& Barbie, D. A. Inflammation as a driver and vulnerability of KRAS mediated oncogenesis. Semin Cell Dev Biol 58, 127-135, https://doi.org/10.1016/j.semcdb.2016.06.009 (2016).

43. Oner, M. G. et al. Combined Inactivation of TP53 and MIR34A Promotes Colorectal Cancer Development and Progression in Mice Via Increasing Levels of IL6R and PAI1. Gastroenterology 155, 1868-1882, https://doi.org/10.1053/j.gastro.2018.08.011 (2018).

44. Paladini, L. et al. Targeting microRNAs as key modulators of tumor immune response. J Exp Clin Cancer Res 35, 103, https://doi. org/10.1186/s13046-016-0375-2 (2016).

45. Chuvin, N. et al. Acinar-to-Ductal Metaplasia Induced by Transforming Growth Factor Beta Facilitates KRAS(G12D)-driven Pancreatic Tumorigenesis. Cell Mol Gastroenterol Hepatol 4, 263-282, https://doi.org/10.1016/j.jcmgh.2017.05.005 (2017).

46. Liu, J. et al. TGF-beta1 promotes acinar to ductal metaplasia of human pancreatic acinar cells. Sci Rep 6, 30904, https://doi. org/10.1038/srep30904 (2016).

47. Akanuma, N. et al. Paracrine Secretion of Transforming Growth Factor beta by Ductal Cells Promotes Acinar-to-Ductal Metaplasia in Cultured Human Exocrine Pancreas Tissues. Pancreas 46, 1202-1207, https://doi.org/10.1097/MPA.0000000000000913 (2017).

48. Qiao, P. et al. microRNA-34a inhibits epithelial mesenchymal transition in human cholangiocarcinoma by targeting Smad4 through transforming growth factor-beta/Smad pathway. BMC Cancer 15, 469, https://doi.org/10.1186/s12885-015-1359-x (2015).

49. Huang, G. et al. MiRNA-34a reversed TGF-beta-induced epithelial-mesenchymal transition via suppression of SMAD4 in NPC cells. Biomed Pharmacother 106, 217-224, https://doi.org/10.1016/j.biopha.2018.06.115 (2018).

50. Genovese, G. et al. microRNA regulatory network inference identifies miR-34a as a novel regulator of TGF-beta signaling in glioblastoma. Cancer Discov 2, 736-749, https://doi.org/10.1158/2159-8290.CD-12-0111 (2012).

51. Liou, G. Y. et al. Macrophage-secreted cytokines drive pancreatic acinar-to-ductal metaplasia through NF-kappaB and MMPs. J Cell Biol 202, 563-577, https://doi.org/10.1083/jcb.201301001 (2013).

52. Siveke, J. T. \& Crawford, H. C. KRAS above and beyond - EGFR in pancreatic cancer. Oncotarget 3, 1262-1263, https://doi. org/10.18632/oncotarget.750 (2012)

53. Maier, H. J. et al. Requirement of NEMO/IKKgamma for effective expansion of KRAS-induced precancerous lesions in the pancreas. Oncogene 32, 2690-2695, https://doi.org/10.1038/onc.2012.272 (2013).

54. Ling, J. et al. KrasG12D-induced IKK2/beta/NF-kappaB activation by IL-1alpha and p62 feedforward loops is required for development of pancreatic ductal adenocarcinoma. Cancer Cell 21, 105-120, https://doi.org/10.1016/j.ccr.2011.12.006 (2012).

55. Lesina, M. et al. Stat3/Socs3 activation by IL-6 transsignaling promotes progression of pancreatic intraepithelial neoplasia and development of pancreatic cancer. Cancer Cell 19, 456-469, https://doi.org/10.1016/j.ccr.2011.03.009 (2011).

56. Fukuda, A. et al. Stat 3 and MMP7 contribute to pancreatic ductal adenocarcinoma initiation and progression. Cancer Cell 19, 441-455, https://doi.org/10.1016/j.ccr.2011.03.002 (2011).

57. Corcoran, R. B. et al. STAT3 plays a critical role in KRAS-induced pancreatic tumorigenesis. Cancer Res 71, 5020-5029, https://doi. org/10.1158/0008-5472.CAN-11-0908 (2011).

58. Jiang, P. et al. MiR-34a inhibits lipopolysaccharide-induced inflammatory response through targeting Notch1 in murine macrophages. Exp Cell Res 318, 1175-1184, https://doi.org/10.1016/j.yexcr.2012.03.018 (2012).

59. Li, H., Rokavec, M. \& Hermeking, H. Soluble IL6R represents a miR-34a target: potential implications for the recently identified IL-6R/STAT3/miR-34a feed-back loop. Oncotarget 6, 14026-14032, https://doi.org/10.18632/oncotarget.4334 (2015).

60. Weng, Y. S. et al. MCT-1/miR-34a/IL-6/IL-6R signaling axis promotes EMT progression, cancer stemness and M2 macrophage polarization in triple-negative breast cancer. Mol Cancer 18, 42, https://doi.org/10.1186/s12943-019-0988-0 (2019).

61. Gao, F., Liang, B., Reddy, S. T., Farias-Eisner, R. \& Su, X. Role of inflammation-associated microenvironment in tumorigenesis and metastasis. Curr Cancer Drug Targets 14, 30-45 (2014).

62. Nakhai, H. et al. Ptfla is essential for the differentiation of GABAergic and glycinergic amacrine cells and horizontal cells in the mouse retina. Development 134, 1151-1160, https://doi.org/10.1242/dev.02781 (2007).

63. Reichert, M., Rhim, A. D. \& Rustgi, A. K. Culturing primary mouse pancreatic ductal cells. Cold Spring Harb Protoc 2015, 558-561, https://doi.org/10.1101/pdb.prot078279 (2015).

64. Lubeseder-Martellato, C. Isolation, Culture and Differentiation of Primary Acinar Epithelial Explants from Adult Murine Pancreas. BioProtocol 3 (2013).

65. Jochheim, L. S. et al. The neuropeptide receptor subunit RAMP1 constrains the innate immune response during acute pancreatitis in mice. Pancreatology 19, 541-547, https://doi.org/10.1016/j.pan.2019.05.455 (2019).

66. Gress, T. M. et al. A pancreatic cancer-specific expression profile. Oncogene 13, 1819-1830 (1996)

67. Knier, B. et al. Myeloid-derived suppressor cells control B cell accumulation in the central nervous system during autoimmunity. Nat Immunol 19, 1341-1351, https://doi.org/10.1038/s41590-018-0237-5 (2018).

68. Macosko, E. Z. et al. Highly Parallel Genome-wide Expression Profiling of Individual Cells Using Nanoliter Droplets. Cell 161, 1202-1214, https://doi.org/10.1016/j.cell.2015.05.002 (2015).

69. Love, M. I., Huber, W. \& Anders, S. Moderated estimation of fold change and dispersion for RNA-seq data with DESeq2. Genome Biol 15, 550, https://doi.org/10.1186/s13059-014-0550-8 (2014).

70. Subramanian, A. et al. Gene set enrichment analysis: a knowledge-based approach for interpreting genome-wide expression profiles. Proc Natl Acad Sci USA 102, 15545-15550, https://doi.org/10.1073/pnas.0506580102 (2005).

\section{Acknowledgements}

We would like to thank Annett Dannemann, Mathilde Neuhofer, Lina Staufer, Olga Seelbach and Katharina Schulte for expert technical assistance. As well as, Jiang Longchang, Matjaz Rokavec and Gülfem Öner for scientific input. This project was supported by the Emmy Noether Program (FI 1719/2-1) to GVF and the Deutsche Forschungsgemeinschaft (DFG, German Research Foundation) - Project-ID 329628492 - SFB 1321 
(S02) to KS. J.T.S. is supported by the German Cancer Consortium (DKTK), the German Cancer Aid (grant no. 70112505; PIPAC consortium) and the DFG (KFO337/SI 1549/3-1).

\section{Author contributions}

Initiation and concept of study: J.S., H.H.; design experiments and conceived experiments: A.H.S. and G.F. performed experiments: A.H.S., C.L.M., S.Z., J.D. and R.Ö. analysed data: A.H.S., C.L.M., T.E. and K.S. wrote manuscript: A.H.S. edited manuscript: A.H.S., C.L.M, R.R., R.S., H. H., J.S. and G.F.

\section{Competing interests}

The authors declare no competing interests.

\section{Additional information}

Supplementary information is available for this paper at https://doi.org/10.1038/s41598-020-66561-1.

Correspondence and requests for materials should be addressed to G.v.F.

Reprints and permissions information is available at www.nature.com/reprints.

Publisher's note Springer Nature remains neutral with regard to jurisdictional claims in published maps and institutional affiliations.

(c) (i) Open Access This article is licensed under a Creative Commons Attribution 4.0 International License, which permits use, sharing, adaptation, distribution and reproduction in any medium or format, as long as you give appropriate credit to the original author(s) and the source, provide a link to the Creative Commons license, and indicate if changes were made. The images or other third party material in this article are included in the article's Creative Commons license, unless indicated otherwise in a credit line to the material. If material is not included in the article's Creative Commons license and your intended use is not permitted by statutory regulation or exceeds the permitted use, you will need to obtain permission directly from the copyright holder. To view a copy of this license, visit http://creativecommons.org/licenses/by/4.0/.

(c) The Author(s) 2020 